\title{
TRANSMISSIBLE SILVER RESISTANCE READILY EVOLVES IN HIGH-RISK CLONE ISOLATES OF KLEBSIELLA PNEUMONIAE
}

\author{
AdRIENN HANCZVIKKEL ${ }^{1 *}$, MIKLÓS FÜZI $^{2}$, ERIKA UNGVÁRI $^{3}$ and ÁKOS TÓTH ${ }^{3}$ \\ ${ }^{1}$ Doctoral School on Material Sciences and Technologies, Óbuda University, \\ Budapest, Hungary \\ ${ }^{2}$ Institute of Medical Microbiology, Semmelweis University, Budapest, Hungary \\ ${ }^{3}$ Division of Bacteriology, Mycology, Parasitology and Typing, National Public Health \\ Institute, Budapest, Hungary
}

(Received: 10 April 2018; accepted: 9 May 2018)

\begin{abstract}
Silver is used extensively in both hospitals and outpatient clinics as a disinfectant coating agent on various devices. Resistance to silver was recently reported as an emerging problem in Enterobacteriaceae. Multidrug-resistant highrisk clones of Klebsiella pneumoniae are common causes of serious healthcareassociated infections worldwide posing a serious threat to patients. In this study, we investigated the capacity of both high-risk (CG14/15 and CG258) and minor clone strains of $K$. pneumoniae to develop resistance to silver. Resistance was induced in vitro in silver-susceptible but otherwise multidrug-resistant clinical isolates. Genetic alterations in the silver-resistant derivative strains with regard to the silver-susceptible isolates were investigated by whole-genome sequencing. The transferability of highlevel resistance to silver was also tested. We demonstrated that the high-level resistance to silver can quickly evolve as a consequence of a single-point mutation either in the cus $\mathrm{S}$ gene of the chromosomally encoded CusCFBARS efflux system and/or in the silS gene of the plasmid-encoded Copper Homeostasis and Silver Resistance Island (CHASRI) coding also for a metallic efflux. The minimal inhibitory concentrations (MICs) of the strains increased from $4 \mathrm{mg} / \mathrm{L}(23.5 \mu \mathrm{M}) \mathrm{AgNO}_{3}$ to $>8,500 \mathrm{mg} / \mathrm{L}(>50,000 \mu \mathrm{M}) \mathrm{AgNO}_{3}$ during induction. Harboring the CHASRI proved an important selective asset for K. pneumoniae when exposed to silver. Successful conjugation experiments using Escherichia coli K12 J5-3 ${ }^{\text {Rif }}$ as recipient showed that high-level silver resistance can transmit between strains of high-risk clones of $K$. pneumoniae (ST15 and ST11) and isolates from additional species of Enterobacteriaceae. The lack of fitness cost associated with the carriage of the CHASRI in a silver-free environment and the presence of the RelEB toxin-antitoxin system on the conjugative plasmids could advance the dissemination of silver resistance. Our results show that multidrug-resistant high-risk clones of $K$. pneumoniae are capable of evolving and transmitting high-level resistance to
\end{abstract}

*Corresponding author; E-mail: hanczvikkel.a@gmail.com 
silver. This observation should warrant a more judicious use of silver coated-devices to prevent the extensive dissemination of silver resistance.

Keywords: silver resistance, CHASRI, Klebsiella pneumoniae, high-risk clone, multidrug resistance

\section{Introduction}

Klebsiella pneumoniae is a primarily opportunistic pathogen capable of causing severe healthcare-associated infections. Neonates, elders, and immunocompromised patients are at greater risk. K. pneumoniae is one of the six "ESKAPE bugs" - the key pathogens (Enterococcus faecium, Staphylococcus aureus, K. pneumoniae, Acinetobacter baumannii, Pseudomonas aeruginosa, and Enterobacter species) - which compromise the healthcare provision [1]. The treatment of K. pneumoniae infections has been complicated by the emergence of extensive antimicrobial resistance (AMR). In the past two decades, several important, horizontally spreading AMR genes were detected in this species: extended-spectrum $\beta$-lactamases (ESBLs), such as SHV- or CTX-M-type; carbapenemases, such as KPC, NDM, VIM, or OXA-48-like; and the plasmid-borne colistin resistance gene ( $m c r-1)$ [2].

While AMR can occur across a broad range of $K$. pneumoniae clones, only a few of them have become globally distributed. About $72 \%$ of all reported outbreaks were caused by one of the following five common clonal groups (CG258, CG14/15, CG17/20, CG43, and CG147) [2]. In 2016, CG258 and CG14/15 were the most prevalent CGs causing outbreaks all over the world. CG258 (main sequence types: ST11 and ST258) is undoubtedly the most widely recognized and globally distributed clonal group [2]. ST11 is a pandemic K. pneumoniae clone, an evolutionary precursor of ST258, and it is responsible for many outbreaks primarily in Europe and Asia. ST258 is a highly adaptive epidemic clone and is the major cause of carbapenem-resistant K. pneumoniae infections [2]. While the CG258 clonal group harbors a diverse arsenal of carbapenemases and ESBL genes (KPC, NDM-1, OXA-48, and CTX-M-15), the ST258 clone is predominantly associated with the KPC-2 and KPC-3 carbapenemases [3]. CG14/15 is the other globally distributed clonal group of $K$. pneumoniae, which has also been associated with a diverse selection of important $\beta$-lactamase genes (CTX-M-15, KPC, NDM-1, OXA-48, OXA-181, and VIM-1) [2].

The effectiveness of antibiotics has been severely compromised by the emergence of multidrug- or pandrug-resistant pathogens. With the increase of antibiotic resistance other alternative antimicrobial agents have gained increased attention in the past decades to prevent infections and silver compounds that 
belong to the most popular ones. Silver has been used as an important antimicrobial agent throughout recorded history [4]. Nowadays, it is widely applied in hospitals (to treat burns, wounds, and ulcers, to coat indwelling medical devices to inhibit microbial colonization and biofilm development, in dental-amalgam and bone-cement, etc. [5, 6]) and in daily life (deodorants, underwear, sport and bed clothing, supermarket-available colloidal "silver-gelatinate" for washing salad, domestic water purification cartridges, etc. [7]), as well. Silver cations are usually microbicidal at low concentrations without adverse effects for humans. $\mathrm{Ag}^{+}$ions have multiple target sites within the bacterial cell; they attach to the cell membrane or envelope, destroy the membrane or get inside the pathogen through transmembrane proteins, bind to thiol groups $(-\mathrm{SH})$ in key enzymes of ATP production, denature enzymes, inactivate them, or intercalate between the base pairs of DNA, disrupting the hydrogen bonding $[8,9]$.

Protection against the toxicity of heavy metals remains essential for all bacteria, thus sophisticated, intricate, and precisely regulated systems have evolved to maintain homeostasis. Due to the structure of their cell wall, Gram-negative bacteria need to deal with both cytoplasmic and periplasmic heavy metals. Several mechanisms such as intra- and extracellular sequestration, enzymatic detoxification, and active efflux can protect the bacteria from the toxicity of metals [10]. The main role of these native systems is to maintain the homeostasis of copper, which is an essential, but potentially toxic micronutrient. Copper and silver share very similar chemical- and ligand-binding properties; thus, systems that maintain copper homeostasis can also actively detoxify silver. The chromosomally encoded CusCFBARS system comprises of a tricomponent RND efflux system (CusCBA), a small periplasmic $\mathrm{Cu}$ and $\mathrm{Ag}$ ions binding protein (CusF), and a two-component regulatory system (CusRS) [11-14]. The operon of the efflux pump and that of the regulatory system is transcribed in a back-to-back fashion (Figure 1A). Under the conditions of elevated concentrations of $\mathrm{Cu}$ or $\mathrm{Ag}$ ions, $\mathrm{CusS}$ and CusR are essential for the induction of the copper efflux genes cusCFBA.

Silver/copper resistance genes can also be harbored on plasmids. The Copper Homeostasis and Silver Resistance Island (CHASRI) [11] contains two clusters (Figure 1B), the plasmid-borne copper-resistance system (Pco) and the silver-resistance system (Sil), which has sequence and functional homology with the copper sensing copper efflux system (Cus) [7, 15]. According to Staehlin et al. [11], the CHASRI was derived from a single evolving event by the linkage of the two copper resistance mechanisms, likely in a close relative of Enterobacter cloacae. The emergence of CHASRI could be a response to the previously unprecedented levels of copper stress caused by humans (tools, disinfection, plumbing, animal husbandry, crop protection, and preservation of perishable commodities) [11], but it can also serve for silver detoxification in bacteria. 
A

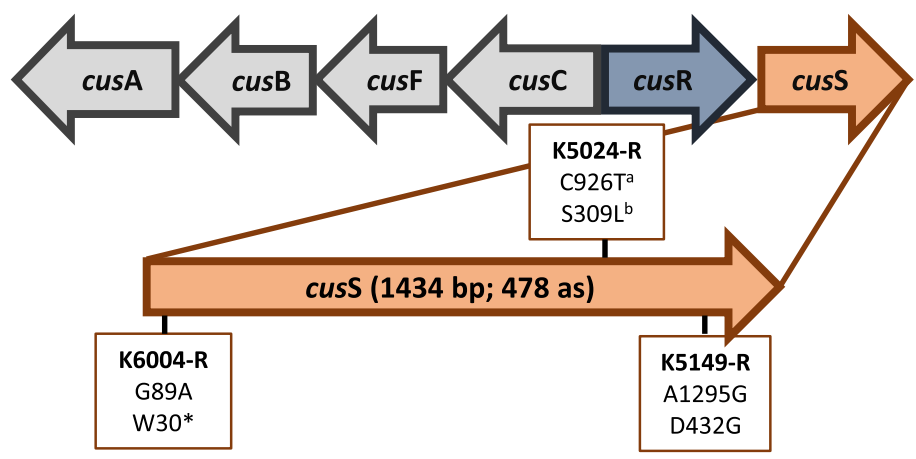

B

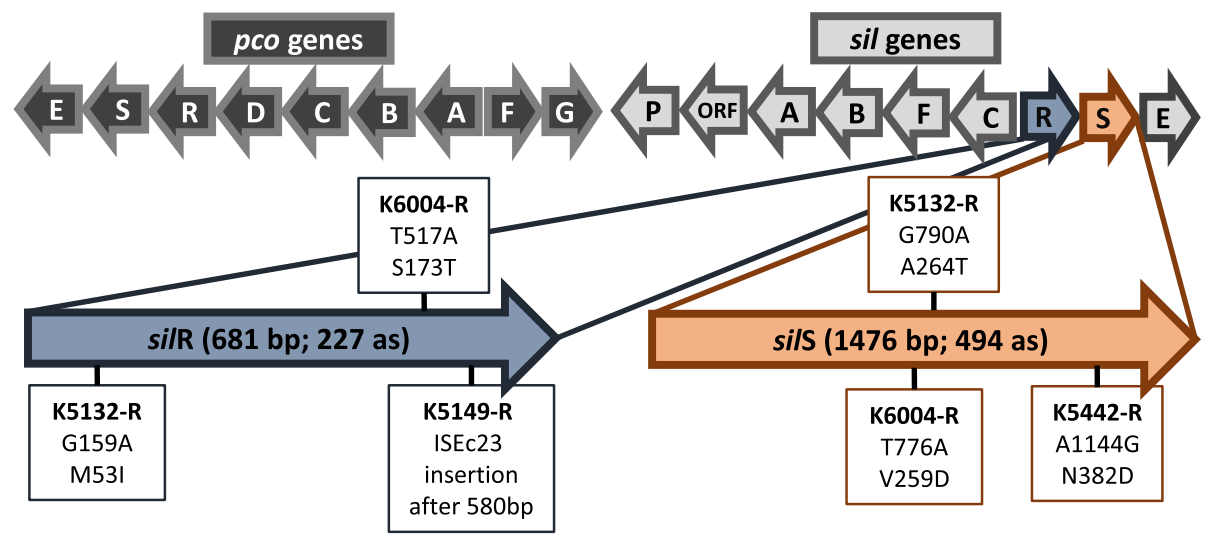

Figure 1. Schematic illustration of the silver efflux systems and the location of detected alterations in silver-resistant derivatives. A: chromosomally encoded CusCFBARS system and B: CHASRI island. Superscripts "a" and "b" mean single nucleotide polymorphism and consequent amino acid substitution, respectively. The symbol "*” means stop codon

Since its assembly, CHASRI has dispersed spottily by horizontal gene transfer among Enterobacteriaceae and other families of bacteria. The components of different chromosomally and plasmid-borne metal-resistance systems can cooperate, as they complement each other [10].

The carriage of these native heavy metal homeostasis systems does not basically affect the heavy metal sensitivity of bacteria, but even a single mutation event can lead to high-level resistance against both copper and silver $[11,16]$. Silver-resistant Escherichia coli was already reported in 1969 [17]. Since then, silver-resistant bacteria have repeatedly been isolated from clinical environments, burn wounds, and teeth $[13,18]$. Due to the increasing use of silver for medical 
and non-medical applications, concerns have been raised about the development and spread of silver resistance.

The emergence and spread of heavy metal resistance could endanger the medical use of silver and copper; therefore, further investigations are necessary to better understand the phenomenon. It is especially important in the high-risk clones of $K$. pneumoniae, since treatment opportunities in infections caused by them have already been limited due to high AMR resistance.

The aim of this study was to determine the silver susceptibility of $K$. pneumoniae strains of both high-risk and minor clones and to investigate their inducibility of silver resistance using continuous exposure of subinhibitory concentrations of silver nitrate. The molecular mechanisms of silver resistance were established by whole-genome sequencing and the associated fitness cost was also investigated. The transferability of silver resistance was studied by mating out assays.

\section{Materials and Methods}

\section{Bacterial strains}

The investigated strain collection consisted of seven healthcare-associated, multidrug-resistant $K$. pneumoniae isolates originating from different areas of Hungary between 1998 and 2014 (Table I). We examined five strains from highrisk global clonal groups (CG) (CG14/15 and CG258) and two strains from minor clones (ST25 and ST274). The strains harbored a diverse array of $\beta$-lactamase genes with clinical and epidemiological importance representing the predominant multidrug-resistant K. pneumoniae clones (Table I).

\section{Silver-susceptibility test}

The minimum inhibitory concentration (MIC) values were determined for $\mathrm{AgNO}_{3}$ solution by twofold serial dilution in Mueller-Hinton broth, according to the EUCAST broth microdilution method [19]. According to Sütterlin et al. [6], bacteria with a silver nitrate MIC $>512 \mathrm{mg} / \mathrm{L}$ were classified as silver-resistant.

\section{Inducing silver resistance}

Passage experiments were performed by continuous exposure to increasing concentrations of $\mathrm{AgNO}_{3}$, according to the method by Tóth et al. [20]. The obtained silver-resistant mutants were compared by pulsed-field gel 


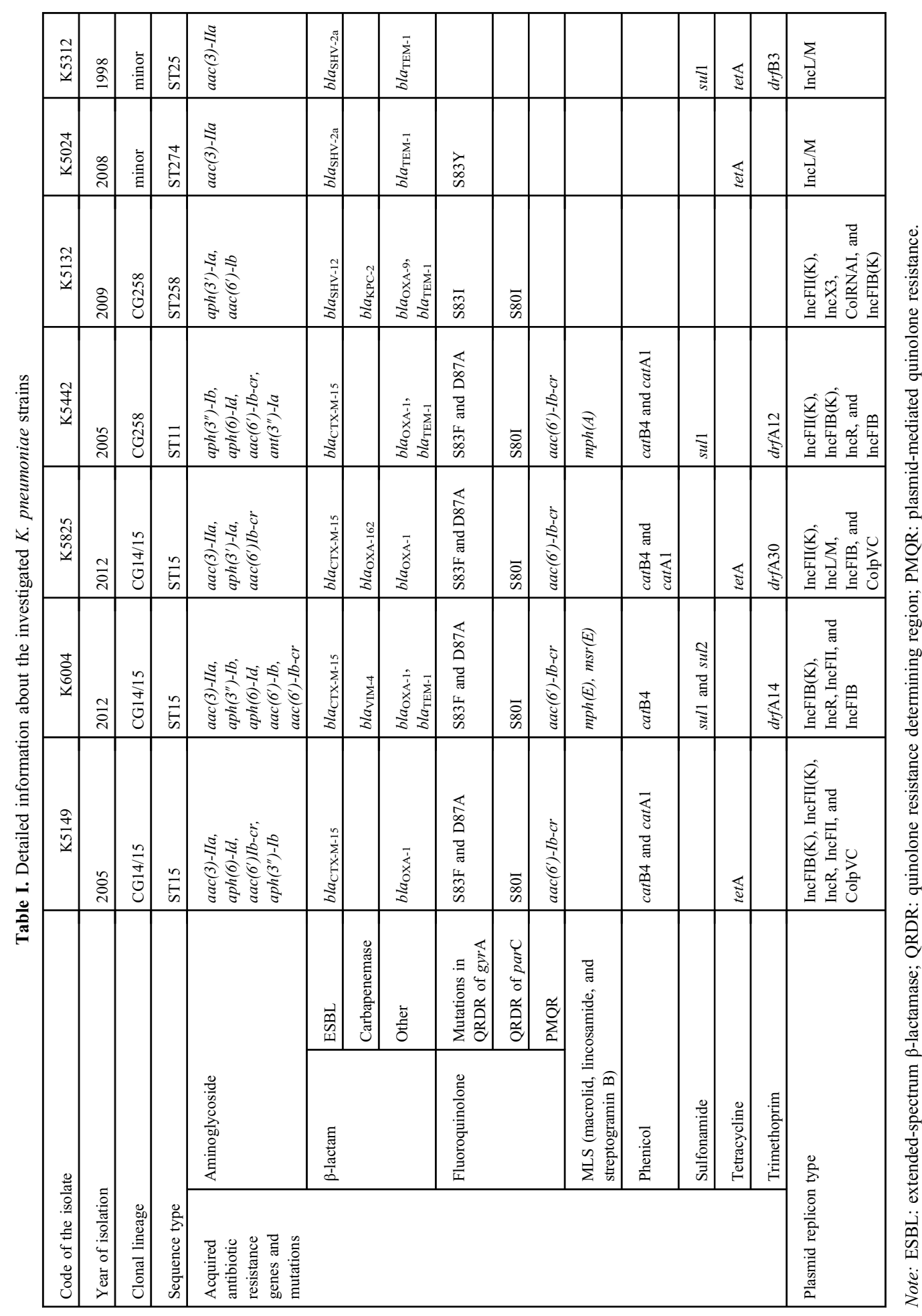


electrophoresis (PFGE) with the original isolates. PFGE was performed in line with the standardized Centers for Disease Control and Prevention protocol [20]. The stability of the silver resistance was examined by subculture of the derivatives onto blood agar plates without silver. We measured the MIC values of the isolates after every 5 th streaking.

\section{Conjugation experiments}

Conjugation experiments were performed by the filter mating procedure, according to the modified method of Werner et al. [21]. CHASRI harboring K5149-R, K6004-R, K5132-R, and K5442-R were used as donor strains and E. coli $\mathrm{K} 12 \mathrm{~J} 5-3^{\mathrm{Rif}}$ was used as recipient strain. Selective agar plates contained $32 \mathrm{mg} / \mathrm{L}(188 \mu \mathrm{M}) \mathrm{AgNO}_{3}$ and $300 \mathrm{mg} / \mathrm{L}$ rifampicin. All transconjugants were confirmed by species identification using MALDI-TOF and $\mathrm{AgNO}_{3}$ susceptibility testing. Plasmid DNA from the donor strains and from transconjugants were obtained using the alkaline lysis method [22] and plasmid profile typing was performed in vertical agarose gels.

\section{Determination of growth rates}

Relative changes in the fitness of bacterial strains were determined in propagation assays. All strains were tested three times and the results were averaged. In vitro planktonic growth rates were measured for the parent strains and silver-resistant derivatives in monocultures with three different concentrations of silver nitrate $\left(0,5\right.$, and $\left.315 \mu \mathrm{M} \mathrm{Ag}^{+}\right)$. Briefly, we adjusted the density of each bacterial suspension in brain-heart infusion broth (BHI; National Public Health Institute, Budapest, Hungary) supplemented by corresponding $\mathrm{Ag}^{+}$ion content to $0.5 \mathrm{McF}$ arland (approximately $10^{8} \mathrm{CFU} / \mathrm{ml}$ ) using a McFarland turbidity meter. The working solutions were obtained after a 100-fold dilution. An amount of $200 \mu \mathrm{l}$ of working solutions were pipetted into microtiter plates. Cultures were incubated at $35^{\circ} \mathrm{C}$ with continuous shaking $(260 \mathrm{rpm})$. Bacterial growth was measured after every $45 \mathrm{~min}$ by recording the absorbance at $620 \mathrm{~nm}$ using a SpectraMax 340 spectrophotometer (Molecular Devices, Sunnyvale, CA). Areas under curve (AUC) were computed to compare the relative fitness of the isolates.

\section{Next-generation sequencing}

We investigated the molecular mechanisms of silver resistance by next generation sequencing of the parent strains' and derivatives' bacterial genomes. 
The genomic DNA extraction was performed using UltraClean Microbial DNA isolation kit (MO BIO Laboratories, USA) following the manufacturer's instructions. The DNA of plasmids from transconjugants was extracted by the alkaline lysis method and precipitated by ethanol [22]. Sequencing libraries were prepared with SureSelect QXT Library Prep Reagent Kit (Agilent Technologies, USA) according to the manufacturer's instructions. Sequencing was performed on a MiSeq system using the MiSeq reagent Kit v2 (Illumina, San Diego, CA, USA) generating 250-bp paired-end reads. Raw reads were processed using online tools of Illumina BaseSpace, which is a cloud-based genomics analysis and storage platform. We used FASTQC for quality control of sequencing, FASTQ toolkit for quality trimming, and SPAdes Genome Assembler 3.9.0. for de novo genome assembly. For de novo assembly of plasmids, plasmidSPAdes Genome Assembler 3.9.0. was applied. Assembled genomes were analyzed using online bioinformatics tools of Center for Genomic Epidemiology, such as ResFinder [23], MyDbFinder, PlasmidFinder [24], pMLST [24] (https://cge.cbs.dtu.dk/services/; Technical University of Denmark), and the plasmid MLST website (https:// pubmlst.org/plasmid/; developed by Keith Jolley and sited at the University of Oxford [25]) and compared with GenBank data using BLAST (http://blast. ncbi.nlm.nih.gov/Blast.cgi) and to each other by SeqSphere+ (Ridom GmbH, Germany) using core and accessory genome gene sets. The analysis of CHASRIs was performed using SnapGene Viewer 3.3.3 software. Integron structure has been named according to INTEGRALL database (http://integrall.bio.ua.pt).

\section{Results}

Parent strains: Silver efflux systems and $\mathrm{AgNO}_{3} \mathrm{MIC}$ values

The antibiotic resistant determinants carried by our isolates were investigated previously [20,26-30] or by whole-genome sequencing and are summarized in Table I.

Table II and Figure 1 show the silver efflux systems of the examined K. pneumoniae strains. Chromosomally encoded cusCFBASR system was found in all the seven strains, but only four of them harbored the CHASRI on plasmid (Figure 1 and Table II). Two minor clone strains (K5024: ST274 and K5312: ST25) and the K5825 (ST15) isolate did not carry CHASRI. The structure of CHASRI was identical in all of the K. pneumoniae strains regardless of their sequence types (Figure 1B).

The $\mathrm{AgNO}_{3}$ MIC values of the seven parent strains proved similar (Table II) regardless of sequence types or CHASRI carriage. All of the strains showed a silver-sensitive phenotype. 


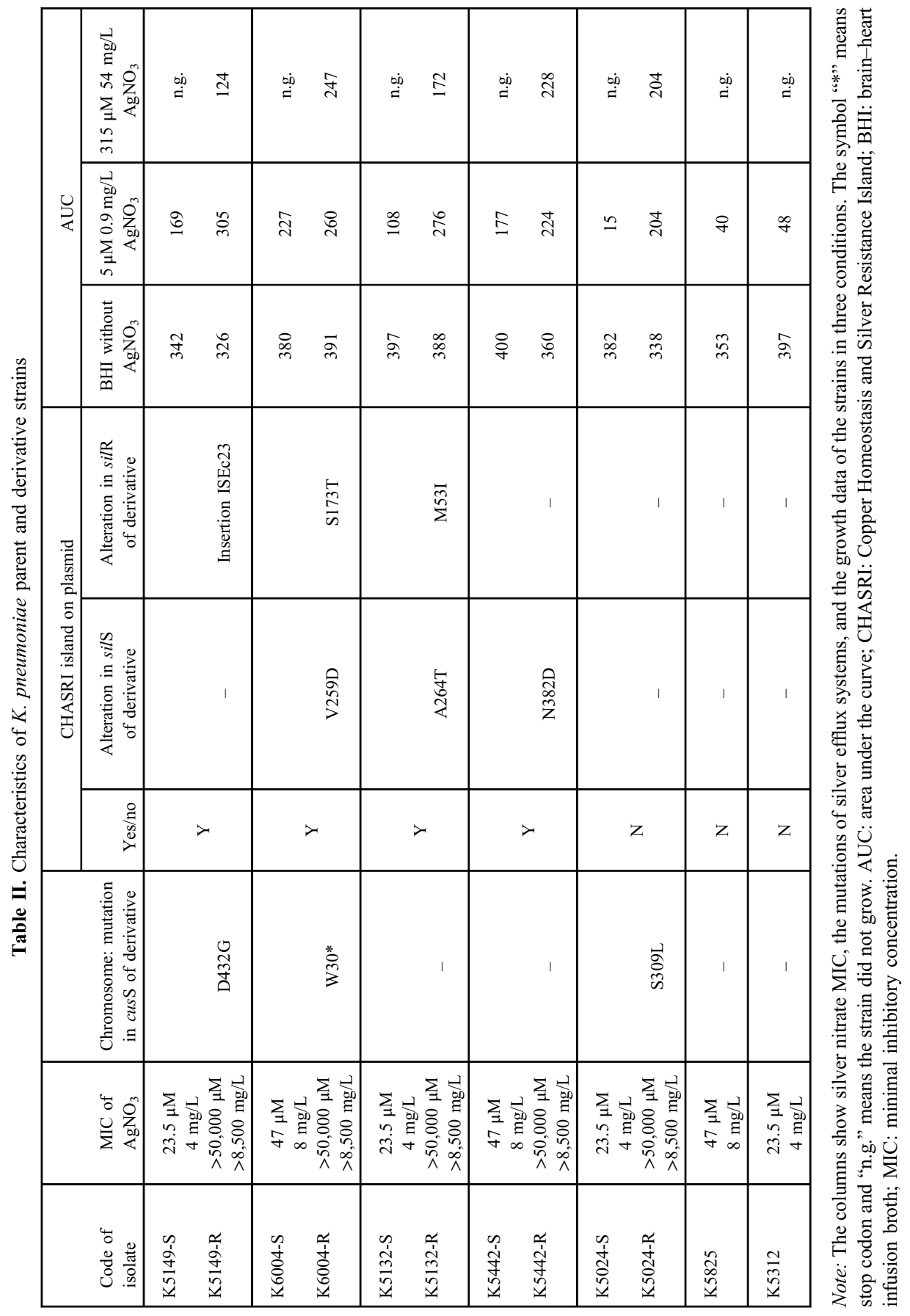


Results of passage experiment, $\mathrm{AgNO}_{3} \mathrm{MIC}$ values of silver-resistant derivative strains

Resistance to silver could be induced in five of our seven strains (71\%). Four of them harbored the CHASRI (Table II). Among the five silver-resistant derivatives, two belonged to ST15, two to CG258 (ST11 and ST258), and one to the ST25 sequence type (Table II). The silver-resistant derivatives of K. pneumoniae strains proved to be indistinguishable by PFGE from the original parent isolates (data not shown).

All silver-resistant derivatives were able to proliferate in broth with an $\mathrm{AgNO}_{3}$ concentration $>8,500 \mathrm{mg} / \mathrm{L}\left(>50,000 \mu \mathrm{M} \mathrm{Ag}^{+}\right)$(Table II). The silverresistant phenotype remained stable after 25 subcultures in the absence of silver.

Mutations in silver efflux systems

The sequencing data of the parent strains and the derivatives were compared. Table II and Figure 1 show different mutations of the strains in chromosomally encoded cus $\mathrm{S}$ and in plasmid encoded silS, silR genes of CHASRI. We found different missense mutations in three distinct genes, one nonsense mutation in cus S, and one insertion of an insertion sequence element in silR gene. No differences in any of the pco genes were detected. The nucleotide sequences of CHASRIs from K5149-S/R, K6004-S/R, K5132-S/R, and K5442-S/R strains have been deposited in GenBank under accession numbers from MH130217 to MH130224.

\section{Growth rate of parent and silver-resistant derivative strains}

Figure 2 shows the growth curves of our isolates and Table II displays the calculated AUC values. Without silver, the parent strains showed similar growth curves and AUC values. In the presence of $0.9 \mathrm{mg} / \mathrm{L} \mathrm{AgNO}_{3}(5 \mu \mathrm{M}$, sub-MIC concentration), the AUC values of parent strains were reduced, the exponential phase of their growth delayed compared with those in silver-free BHI. Furthermore, in the presence of silver in sub-MIC concentration, the growth rates of the parent strains differed from each other (Figure 2). The three strains that did not carry CHASRI showed the lowest AUC values and the biggest delay in starting of exponential growth phase. In $54 \mathrm{mg} / \mathrm{L}\left(315 \mu \mathrm{M}\right.$, supra-MIC) concentration of $\mathrm{AgNO}_{3}$, the parent strains were not able to grow.

The derivative strains showed similar AUC values in BHI without silver nitrate (Table II). The presence of silver slightly reduced their AUC values due to the 

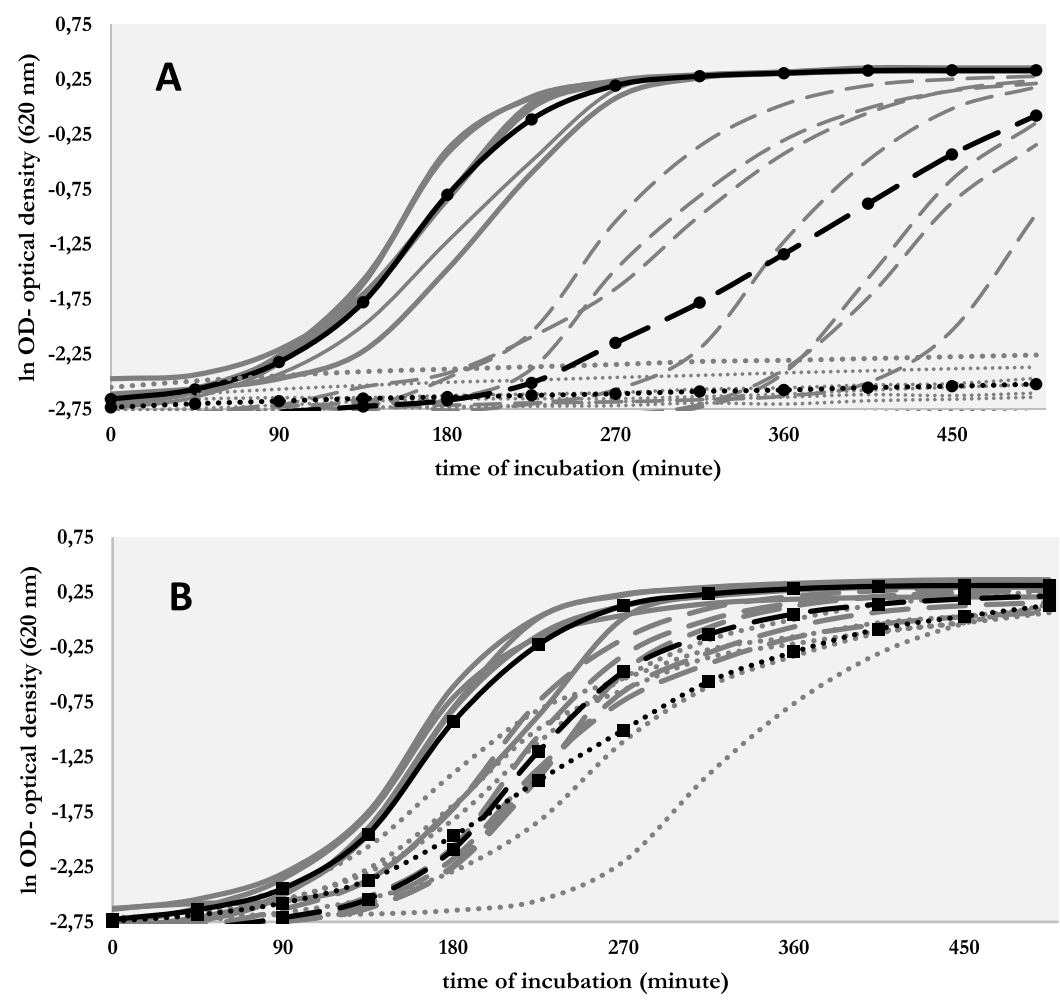

Figure 2. Growth curves of (A) parent strains and (B) silver-resistant derivatives in brain-hearth infusion with different concentration of silver nitrate [continuous line: without silver, dashed line: $5 \mu \mathrm{M} \mathrm{Ag}^{+}\left(0.9 \mathrm{mg} / \mathrm{L} \mathrm{AgNO}_{3}\right)$, and dotted line: $\left.315 \mu \mathrm{M} \mathrm{Ag}^{+}\left(54 \mathrm{mg} / \mathrm{L} \mathrm{AgNO}_{3}\right)\right]$. Gray curves show the individual results of the strains and black curves show the averaged values

delay in starting the exponential growth phase (Figure 2), but the growth rates were not always associated with the concentration of silver. The growth curves recorded in solutions with 5 and $315 \mu \mathrm{M}$ of $\mathrm{Ag}^{+}$overlap each other, with the exception of K5149-R. The higher silver concentration substantially delayed the growth of K5149-R, but finally this strain reached the same optical density as those growing in silver-free BHI.

In a silver nitrate-free environment, the AUC values of the parent strains and of their silver-resistant derivatives remained similar to each other (Table II); thus, the derivatives did not suffer fitness cost due to the developing high-level silver resistance. In the presence of silver, the derivatives showed unequivocal growth advantage relative to the parent strains. 
Results of conjugation

Conjugation was successful in case of K6004-R and K5442-R K. pneumoniae strains, which belonged to the widespread ST15 and ST11 sequence types, respectively. The $\mathrm{AgNO}_{3}$ MIC values of the recipient E. coli $\mathrm{K} 12 \mathrm{~J}-53^{\mathrm{Rif}}$ and both the transconjugants (TR6004 and TR5442) were $4(23.5 \mu \mathrm{M})$ and $8,500 \mathrm{mg} / \mathrm{L}\left(50,000 \mu \mathrm{M} \mathrm{Ag}^{+}\right)$, respectively. Plasmids obtained from TR6004 and TR5442 were different [p6004 - size: $197 \mathrm{kbp}$, replicon types: IncFII and IncFIB(K), pMLST: K9:A-:B-; p5442 - size: $160 \mathrm{kbp}$, replicon types: IncFII(K) and IncFIB(K), pMLST: K1:A-:B-]. A BLAST comparison of p6004 and p69-1 (GenBank: CP025457.1) revealed a $99 \%$ identity (82\% query coverage) between the two plasmids. Plasmid p5442 showed 99\% similarity to pKPN3 (GenBank: CP011577.1) with $92 \%$ query coverage. Compared to pKPN3 plasmid, p5442 harbored additional AMR genes (aadA2, sul1, dfrA12, and catA1), most of them are part of a Class-I integron (structure of In27 integron: $5^{\prime} \mathrm{CS}-\mathrm{dfrA} 12-\mathrm{gcuF}-\mathrm{aadA} 2-\mathrm{qacE} \Delta 1-$ sul1orf5 $\Delta-3^{\prime} \mathrm{CS}$ ). Both plasmids contain RelBE type II toxin-antitoxin (TA) system.

\section{Discussion}

The silver MIC values of the parent $K$. pneumoniae strains were in the same to those reported by Randall et al. [16]. Similar to the study by Sütterlin et al. [6], we found no differences among the silver MIC values of parent strains with diverse genetic backgrounds. The MIC values of silver-resistant derivative strains increased dramatically upon induction (from 4-8 to $>8,500 \mathrm{mg} / \mathrm{L}$ ). Sütterlin et al. [6] detected similarly elevated MIC values $(>512 \mathrm{mg} / \mathrm{L})$ in other species of Enterobacteriaceae. According to Randall et al. [16], the silver resistance mediated by the Sil system had minimal impact on bacterial fitness when compared with silver-susceptible parental strains. Our results not only confirmed this observation but also complement it. In a silver-free environment, chromosomally cusS-mediated silver resistance did not show any fitness cost.

We showed that the development of silver-resistance is not clone-specific in K. pneumoniae. Resistance to silver could be quickly induced in both some highrisk clone strains (ST15, ST258, and ST11) and a minor clone isolate (ST274). In contrast, one of the ST15 strains and the ST25 K. pneumoniae isolate proved unable to evolve resistance to silver. The ability of globally distributed $K$. pneumoniae clones to develop resistance to silver is of concern as they could compromise the efficacy of silver in the healthcare setting.

The silver-resistant derivative of K5024 (ST274) proved that the presence of the CHASRI is not necessarily required for developing silver resistance. 
Sütterlin et al. [6] were already able to induce silver resistance in one sil-negative K. pneumoniae strain, but the genetic mechanism was not investigated. According to Randall et al. [16], upregulation of chromosomally encoded CusCFBA efflux system itself is sufficient to remove silver ions from $E$. coli, provided it is associated with simultaneous loss of OmpC/F porins from the outer membrane. Comparing the genetic data of K5024-S and K5024-R, we found a missense mutation in cusS gene (Table II) and a nonsense mutation in ompK36 gene (Q261*, where * means stop codon), which is homolog to the ompC gene in E. coli [31]. These results confirm the conclusions of Randall et al. and prove that such combination of mutation events can also occur in K. pneumoniae.

Although the carriage of the CHASRI is not a prerequisite for the development of resistance to silver, it clearly promotes the evolvement of resistance. While all four strains carrying the CHASRI were able to attain high MIC values for silver, only one of the three isolates void of this genetic element proved capable of achieving similarly high level of resistance.

Our results are in agreement with the findings of Sütterlin et al. [6] obtained with silS-positive Enterobacteriaceae isolates. In 12 isolates among the tested 17 strains, they found SNPs concentrated in two segments of the silS gene (629-725 and 919-1054 bp). We suppose that due to the cooperation of chromosomally and plasmid-borne metal homeostasis systems, mutations of both cus S and silS genes can cause constitutive expression of the CusCFBA and SilCFBA efflux transporters. In the K5149-R strain, we identified a missense mutation of cus $\mathrm{S}$, but in K6004-R, K5132-R, and K5442-R strains alterations of silS gene were responsible for silver-resistant phenotype (Figure 1 and Table II). Consequently, harboring silS-encoded plasmid in one or more copies increases the probability of the emergence of suitable mutations.

Strains without CHASRI not only have less tools for developing silver resistance, but also have lower proliferation ability in the presence of silver. In the presence of sub-MIC concentration, $\mathrm{AgNO}_{3} \mathrm{~K} 5024-\mathrm{S}$, K5312, and K5825 strains showed lower and delayed growth compared with strains having CHASRI (Figure 2). We assume that the growth disadvantage is caused by the lower number of expressed membrane efflux pumps, which is the consequence of the missing CHASRI. Our theory is supported by the decreased growth of K5149-R strain in solution of $315 \mu \mathrm{M}$ of $\mathrm{AgNO}_{3}$ compared to BHI containing $5 \mu \mathrm{M}$ of $\mathrm{AgNO}_{3}$ (Table II). The silR response regulator gene of K5149-R strain lost its function due to the insertion of ISEc23 element; thus, the expression of SilCFBA system was decreased, which made the proliferation of the bacterium slower relative to strains carrying a native silR.

In the study of Deshpande and Chopade [32], silver resistance proved to be transferable from $A$. baumannii to $E$. coli $\mathrm{K} 12$ during conjugation, but according to 
our knowledge, this is the first description of successful silver resistance transfer between $K$. pneumoniae and E. coli strains. In this investigation, strains belonging to both ST15 and ST11 K. pneumoniae were able to transmit silver resistance genes on two different plasmids. Analysis of p5442 plasmid showed that the CHASRI was cotransferred with AMR genes. This implies that spread of CHASRI among bacterial species could be helped by selection pressure of antibiotics. Both plasmids contained RelBE3 toxin-antitoxin system, which plays vital role in plasmid maintenance, due to the post-segregational killing effect. The RelBE3 system is composed of a stable toxin (protein RelE, which is capable of cleaving mRNA) and an unstable antitoxin (protein RelB, which neutralizes the toxin by forming a toxin-antitoxin complex) [33]. If the bacterium loses the TA system harboring plasmid, the stable toxin will kill it after the degradation of the unstable antitoxin. Presence of the RelBE system on CHASRI harboring plasmids ensures the maintenance of silver resistance in both donor strains and transconjugants.

In the presence of silver, the carriage of CHASRI could be an important selection asset for bacterial strains due to the higher growth rate and the multiple possibilities to develop a silver-resistant phenotype. According to Sütterlin et al. [6], the presence of sil genes was detected in $41 \%$ of the examined 129 healthcareassociated $K$. pneumoniae isolates. Originally, only $3 \%$ of the isolates showed phenotypic silver resistance, but based on the results of Sütterlin et al. and of this study a single mutation is sufficient to drastically elevate the silver MIC values. Our results proved that silver-resistant phenotype could be very stable and transferable by conjugation. All these observations emphasize the need for a prudent use of silver-containing products.

\section{Conclusions}

The mechanism of silver resistance is usually investigated using E. coli isolates. Although Sütterlin et al. [6] sequenced the whole genome of a ST20 $K$. pneumoniae strain before and after silver exposure, further research is required to understand the development of silver resistance in this species. We investigated the inducibility of silver resistance in seven $K$. pneumoniae strains, belonging to the widespread CG14/15 and CG258 clonal groups or to minor clones applying passage experiments. We also investigated the molecular background of silver resistance by whole-genome sequencing and tested the transferability of silver resistance by mating out assays.

Our findings contribute to the better understanding of the mechanism of silver resistance in high-risk $K$. pneumoniae strains. We proved that silverresistance phenotype can arise after a single-point mutation in either cus $\mathrm{S}$ or 
silS genes, but carriage of the CHASRI could be an important selection advantage for bacterial strains in the presence of silver. The carriage of the CHASRI is not associated with any fitness cost in a silver-free environment. Successful mating out assays proved that plasmid-mediated silver resistance could spread among $K$. pneumoniae and other species. The presence of the RelBE toxin-antitoxin system on the plasmids harboring the CHASRI contributes to the maintenance of the silver-resistant phenotype.

In vivo developed/acquired resistance to silver was already detected among Enterobacteriaceae strains [6, 34]; moreover, commercially available silverimpregnated burn and wound dressings proved to be inefficient against two of these strains [34]. The prevalence of the sil genes in various Enterobacteriaceae species is common in the healthcare setting $[6,35])$. These observations completed by our results draw attention to the prudent use of silver. When treating an infection caused by a $K$. pneumoniae high-risk clone isolate, not only antibiotic resistance but also resistance to silver should be considered. We have shown that a stable, transferable, high-level resistance to silver can readily develop in multidrug-resistant $K$. pneumoniae high-risk clones.

\section{Acknowledgements}

The authors would like to express their sincere thanks to Melinda Jakab, Laura Jánvári, Júlia Topf, and Andrea Torma for their continuous technical assistance.

\section{Conflict of Interest}

The authors declare no conflict of interest. This research did not receive any specific grant from funding agencies in the public, commercial, or not-for-profit sectors.

\section{References}

1. Rice, L. B.: Federal funding for the study of antimicrobial resistance in nosocomial pathogens: No ESKAPE. J Infect Dis 197, 1079-1081 (2008).

2. Wyres, K. L., Holt, K. E.: Klebsiella pneumoniae population genomics and antimicrobialresistant clones. Trends Microbiol 24, 944-956 (2016).

3. Pitout, J. D., Nordmann, P., Poirel, L.: Carbapenemase-producing Klebsiella pneumoniae, a key pathogen set for global nosocomial dominance. Antimicrob Agents Chemother 59, 5873-5884 (2015).

4. Alexander, J. W.: History of the medical use of silver. Surg Infect (Larchmt) 10, 289-292 (2009). 
5. Weber, D. J., Rutala, W. A.: Self-disinfecting surfaces: Review of current methodologies and future prospects. Am J Infect Control 41, 31-35 (2013).

6. Sütterlin, S., Dahlö, M., Tellgren-Roth, C., Schaal, W., Melhus, Å.: High frequency of silver resistance genes in invasive isolates of Enterobacter and Klebsiella species. J Hosp Infect 96, 256-261 (2017).

7. Silver, S.: Bacterial silver resistance: Molecular biology and uses and misuses of silver compounds. FEMS Microbiol Rev 27, 341-353 (2003).

8. Fouda, M. M.: Antibacterial modification of textiles using nanotechnology. In Bobbaralas V. (ed): A search for antibacterial agents. InTech, Rijeka, Croatia, 2012, pp. 47-72.

9. McDonnell, G., Russell, A. D.: Antiseptics and disinfectants: Activity, action, and resistance. Clin Microbiol Rev 12, 147-179 (1999).

10. Bondarczuk, K., Piotrowska-Seget, Z.: Molecular basis of active copper resistance mechanisms in Gram-negative bacteria. Cell Biol Toxicol 29, 397-405 (2013).

11. Staehlin, B. M., Gibbons, J. G., Rokas, A., O’Halloran, T. V., Slot, J. C.: Evolution of a heavy metal homeostasis/resistance island reflects increasing copper stress in enterobacteria. Genome Biol Evol 8, 811-826 (2016).

12. Gudipaty, S. A., Larsen, A. S., Rensing, C., McEvoy, M. M.: Regulation of Cu (I)/Ag (I) efflux genes in Escherichia coli by the sensor kinase CusS. FEMS Microbiol Lett 330, 30-37 (2012).

13. Mijnendonckx, K., Leys, N., Mahillon, J., Silver, S., Van Houdt, R.: Antimicrobial silver: Uses, toxicity and potential for resistance. Biometals 26, 609-621 (2013).

14. Delmar, J. A., Su, C.-C., Yu, E. W.: Bacterial multidrug efflux transporters. Annu Rev Biophys 43, 93-117 (2014).

15. Gupta, A., Matsui, K., Lo, J.-F., Silver, S.: Molecular basis for resistance to silver cations in Salmonella. Nat Med 5, 183-188 (1999).

16. Randall, C. P., Gupta, A., Jackson, N., Busse, D., O’Neill, A. J.: Silver resistance in Gramnegative bacteria: A dissection of endogenous and exogenous mechanisms. J Antimicrob Chemother 70, 1037-1046 (2015).

17. Jelenko, C., 3rd: Silver nitrate resistant E. coli: Report of case. Ann Surg 170, 296-299 (1969).

18. Sütterlin, S., Tano, E., Bergsten, A., Tallberg, A.-B., Melhus, A.: Effects of silver-based wound dressings on the bacterial flora in chronic leg ulcers and its susceptibility in vitro to silver. Acta Derm Venereol 92, 34-39 (2012).

19. EUCAST: Determination of minimum inhibitory concentrations (MICs) of antibacterial agents by broth dilution. Clin Microbiol Infect 9, 9-15 (2003).

20. Tóth, Á., Kocsis, B., Damjanova, I., Kristóf, K., Jánvári, L., Pászti, J., Csercsik, R., Topf, J., Szabó, D., Hamar, P.: Fitness cost associated with resistance to fluoroquinolones is diverse across clones of Klebsiella pneumoniae and may select for CTX-M-15 type extendedspectrum $\beta$-lactamase. Eur J Clin Microbiol Infect Dis 33, 837-843 (2014).

21. Werner, G., Freitas, A. R., Coque, T. M., Sollid, J. E., Lester, C., Hammerum, A. M., Garcia-Migura, L., Jensen, L. B., Francia, M. V., Witte, W.: Host range of enterococcal vanA plasmids among Gram-positive intestinal bacteria. J Antimicrob Chemother 66, 273-282 (2010).

22. Sambrook, J., Russell, D. W.: Molecular cloning: A laboratory manual, 3rd ed. Cold Spring Harbor Laboratory Press, New York, 2001, pp. 2344. 
23. Zankari, E., Hasman, H., Cosentino, S., Vestergaard, M., Rasmussen, S., Lund, O., Aarestrup, F. M., Larsen, M. V.: Identification of acquired antimicrobial resistance genes. J Antimicrob Chemother 67, 2640-2644 (2012).

24. Carattoli, A., Zankari, E., Garcìa-Fernandez, A., Larsen, M. V., Lund, O., Villa, L., Aarestrup, F. M., Hasman, H.: PlasmidFinder and pMLST: In silico detection and typing of plasmids. Antimicrob Agents Chemother 58, 3895-3903 (2014).

25. Jolley, K. A., Maiden, M. C.: BIGSdb: Scalable analysis of bacterial genome variation at the population level. BMC Bioinformatics 11, 595-606 (2010).

26. Jánvári, L., Damjanova, I., Lázár, A., Rácz, K., Kocsis, B., Urbán, E., Tóth, Á.: Emergence of OXA-162-producing Klebsiella pneumoniae in Hungary. Scand J Infect Dis 46, 320-324 (2014).

27. Damjanova, I., Tóth, A., Kenesei, E., Köhalmi, M., Szántai, P., Füzi, M., Pászti, J.: Dissemination of ST274 Klebsiella pneumoniae epidemic clone in newborn and adult hospital settings harbouring SHV-2A or CTX-M-15 type extended spectrum $\beta$-lactamasesproducing known plasmids. Eur J Microbiol Immunol 1, 223-227 (2011).

28. Toth, A., Damjanova, I., Puskás, E., Jánvári, L., Farkas, M., Dobák, A., Böröcz, K., Pászti, J.: Emergence of a colistin-resistant KPC-2-producing Klebsiella pneumoniae ST258 clone in Hungary. Eur J Clin Microbiol Infect Dis 29, 765-769 (2010).

29. Damjanova, I., Tóth, Á., Pászti, J., Hajbel-Vékony, G., Jakab, M., Berta, J., Milch, H., Füzi, M.: Expansion and countrywide dissemination of ST11, ST15 and ST147 ciprofloxacinresistant CTX-M-15-type $\beta$-lactamase-producing Klebsiella pneumoniae epidemic clones in Hungary in 2005 - The new 'MRSAs'? J Antimicrob Chemother 62, 978-985 (2008).

30. Damjanova, I., Tóth, Á., Pászti, J., Jakab, M., Milch, H., Bauernfeind, A., Füzi, M.: Epidemiology of SHV-type $\beta$-lactamase-producing Klebsiella spp. from outbreaks in five geographically distant Hungarian neonatal intensive care units: Widespread dissemination of epidemic R-plasmids. Int J Antimicrob Agents 29, 665-671 (2007).

31. Dutzler, R., Rummel, G., Alberti, S., Hernandez-Alles, S., Phale, P., Rosenbusch, J., Benedi, V., Schirmer, T.: Crystal structure and functional characterization of OmpK36, the osmoporin of Klebsiella pneumoniae. Structure 7, 425-434 (1999).

32. Deshpande, L. M., Chopade, B. A.: Plasmid mediated silver resistance in Acinetobacter baumannii. Biometals 7, 49-56 (1994).

33. Wei, Y.-Q., Bi, D.-X., Wei, D.-Q., Ou, H.-Y.: Prediction of type II toxin-antitoxin loci in Klebsiella pneumoniae genome sequences. Interdiscip Sci Comput Life Sci 8, 143-149 (2016).

34. Finley, P. J., Norton, R., Austin, C., Mitchell, A., Zank, S., Durham, P.: Unprecedented silver resistance in clinically isolated Enterobacteriaceae: Major implications for burn and wound management. Antimicrob Agents Chemother 59, 4734-4741 (2015).

35. Elkrewi, E., Randall, C. P., Ooi, N., Cottell, J. L., O'neill, A. J.: Cryptic silver resistance is prevalent and readily activated in certain Gram-negative pathogens. J Antimicrob Chemother 72, 3043-3046 (2017). 